\title{
Mathematical models of rock mass affected by high pressure gradients
}

\author{
Alexandr Revuzhenko*, and Sergey Lavrikov \\ Chinakal Institute of Mining, Siberian Branch of Russian Academy of Sciences, Novosibirsk, 630091, \\ Russia
}

\begin{abstract}
The authors propose to solve the problems connected with high pressure gradients using a model of a linearly elastic body with a structural parameter. The closed finite difference system of equations is formulated for plain strain deformation conditions. The problem on deformation of rock mass in the vicinity of an underground opening affected by mixedtype gravity-and-tectonic stress field is solved. It is demonstrated that addition of the structure in the solution changes the value of the stress concentration coefficient.
\end{abstract}

\section{Introduction}

Rocks possess very complicated elastoplastic and rheological properties. In some situations, elastic effects prevail, in other circumstances, internal friction, dilatancy, creep, etc. dominate. For this reason, there are no universal mathematical models of rock masses, and the research seems unpractical in this respect. It is expected that universal models can be very complex and, hence, difficultly applicable. The best way is construction of partial models capable to offer sufficiently adequate descriptions of specific situations within an acceptable time and using available facilities $[1,2]$.

The simplest mathematical model of a geomedium is a linear elastic model. It is widely used for solving different problems in geomechanics and is actually a basic model [3-5]. Mechanics of deformable solids advances in two directions. The first direction is connected with various inelastic strains to be included into analysis. And the second direction is development of the basic model of an elastic body. The scope of this research direction encompasses various options of the moment elasticity [6,7], gradient theory [8], etc. [9]. One of the reasons to develop this research trend is the fact that high stress gradients bring uncertainty in the Hooke law, which needs fundamental modification therefore.

Rock mechanics vastly use the results of the first direction research and totally neglects the second direction research findings. In the meanwhile, most problems in geomechanics involve high pressure gradients. Hence, evolution of the basic model along this route seems to be highly relevant.

The basic elastic model rests upon two hypotheses: 1) the Hooke law holds true; 2) the field of displacements is smooth, i.e. has partial derivatives of coordinates. The latter means

\footnotetext{
${ }^{*}$ Corresponding author: revuzhenko@yandex.ru
} 
that local strains in any unit volume of a medium are affine and the displacement field is linear in the coordinate axes.

In real time, rocks contain abundances of pores and fractures of different scales. For this reason, the condition of the displacement field smoothness should be slackened. The related studies [10-12] show that even in case of a linear elastic body, the constitutive equations should include stresses and, also, the second differential coefficients of stresses with respect to coordinates. The dimensionality analysis of rocks immediately picks up the parameter of the length dimension. Consequently, these models can be referred to the class of gradient models with a structural parameter. Let us discuss one of possible implementations of the model [10].

\section{Mathematical model}

In case of plane strain, the differential model [10] reduces to the system of equations below

$$
\begin{gathered}
t_{11}\left(A_{i j}\right)-t_{11}\left(C_{i j}\right)+t_{21}\left(B_{i j}\right)-t_{21}\left(D_{i j}\right)=0, \\
t_{12}\left(A_{i j}\right)-t_{12}\left(C_{i j}\right)+t_{22}\left(B_{i j}\right)-t_{22}\left(D_{i j}\right)=0, \\
t_{12}\left(A_{i j}\right)+t_{12}\left(C_{i j}\right)-t_{21}\left(B_{i j}\right)-t_{21}\left(D_{i j}\right)=0, \\
\frac{u_{1}\left(A_{i j}\right)-u_{1}\left(C_{i j}\right)}{2 r}=\frac{1}{E}\left(\frac{t_{11}\left(A_{i j}\right)+t_{11}\left(C_{i j}\right)}{2}-\mu \frac{t_{22}\left(B_{i j}\right)+t_{22}\left(D_{i j}\right)}{2}\right), \\
\frac{u_{2}\left(B_{i j}\right)-u_{2}\left(D_{i j}\right)}{2 r}=\frac{1}{E}\left(\frac{t_{22}\left(B_{i j}\right)+t_{22}\left(D_{i j}\right)}{2}-\mu \frac{t_{11}\left(A_{i j}\right)+t_{11}\left(C_{i j}\right)}{2}\right), \\
\frac{u_{2}\left(A_{i j}\right)-u_{2}\left(C_{i j}\right)}{2 r}+\frac{u_{1}\left(B_{i j}\right)-u_{1}\left(D_{i j}\right)}{2 r}=\frac{2(1+\mu)}{E} \cdot \frac{t_{12}\left(A_{i j}\right)+t_{12}\left(C_{i j}\right)+t_{21}\left(B_{i j}\right)+t_{21}\left(D_{i j}\right)}{4}, \\
\frac{u_{1}\left(A_{i j}\right)+u_{1}\left(C_{i j}\right)}{2}-\frac{u_{1}\left(B_{i j}\right)+u_{1}\left(D_{i j}\right)}{2}=\xi\left(\frac{t_{11}\left(A_{i j}\right)-t_{11}\left(C_{i j}\right)}{2 r}-\frac{t_{21}\left(B_{i j}\right)-t_{21}\left(D_{i j}\right)}{2 r}\right), \\
-\frac{u_{2}\left(A_{i j}\right)+u_{2}\left(C_{i j}\right)}{2}+\frac{u_{2}\left(B_{i j}\right)+u_{2}\left(D_{i j}\right)}{2}=\xi\left(-\frac{t_{12}\left(A_{i j}\right)-t_{12}\left(C_{i j}\right)}{2 r}+\frac{t_{22}\left(B_{i j}\right)-t_{22}\left(D_{i j}\right)}{2 r}\right) .
\end{gathered}
$$

Here, all displacements and strains are determined at the boundaries of particles with linear size $2 r, i=\overline{1, m}, j=\overline{1, n}$, where $N=m \times n$ is the total number of particles in the computational domain; $E, \mu$ are the Young modulus and Poisson's ratio, respectively; $\xi$ is a structural parameter (Fig. 1). Equations (1) are the equations of equilibrium of a unit volume, the stress tensor components $t_{11}, t_{12}$ are determined on sites marked by a vertical line (at points $A_{i j}, C_{i j}$ ), and the components $t_{21}, t_{22}$ are determined on sites marked by a horizontal line (at points $B_{i j}, D_{i j}$ ). It should be emphasized that these are the classical definitions. Each of the components, for instance, $t_{12}, t_{22}$, represents the vertical component of the force vector (referred to the length of the site). The sites are different, and the notions are different, too, therefore. In continuum models, these components are taken not as one but as two functions to be determined. That is, this circumstance is included in the balance between the number of equations and the number of unknowns. 
Equations (2) describe the Hooke law, while equations (3) are unformulated explicitly in classical elasticity but are actually contained in the assumption of smoothness of the displacement field-postulate of diffeomorphism [13]. Let us discuss this point in more detail. With equations (3) neglected for a time being, system (1), (2) becomes a classical system of finite difference equations of linear elasticity. The vertical displacement $u_{2}$, effective in the horizontal site (points $B_{i j}, D_{i j}$ ), and the vertical displacement $u_{2}$, effective in the vertical site (points $A_{i j}, C_{i j}$ ), are denoted similarly- $u_{2}$. In transition to the continuum model, we have the only unknown function $-u_{2}$. That is to say, the situation is fundamentally different from the description of the stress field and is a consequence of the assumed smoothness of the displacement field.

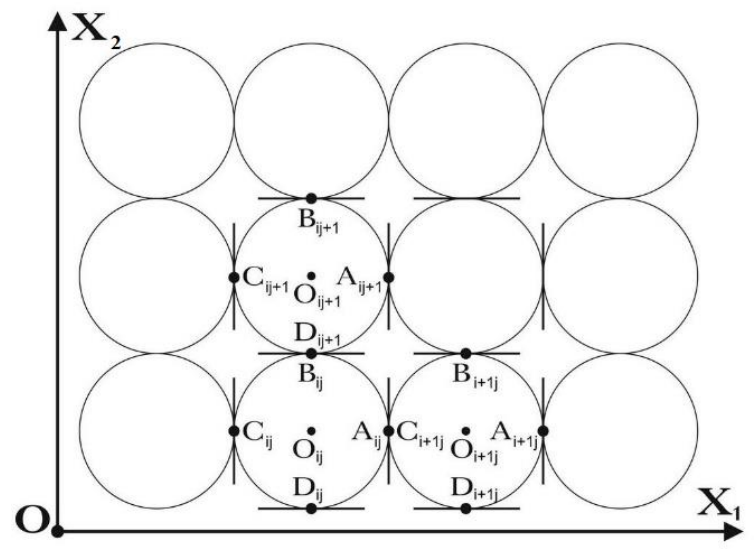

Fig. 1. Internal structure of the medium: configuration of particle contacts.

In our model, the assumption of smoothness is slackened. Accordingly, in transition to the continuum model when $r \rightarrow 0$, for the description of one initial unsmooth field of displacements $\mathbf{u}=\left(u_{1}, u_{2}\right)$, we need to introduce two independent smooth fields: the field $\mathbf{v}=\left(v_{1}, v_{2}\right)$ determined on the vertical sites (at points $\left.A_{i j}, C_{i j}\right)$ and the field $\mathbf{w}=\left(w_{1}, w_{2}\right)$ determined on the horizontal sites (at points $B_{i j}, D_{i j}$ ) (Fig. 1). Consequently, the full symmetry restores in the description of the fields of stresses and displacements! In the end, the difference mesh consists of two nested meshes offset relative to each other, and the displacements and stresses are determined on these meshes [14].

It is readily noticed that the condition $\xi=0$ in the continuum model leads to the equality $\mathbf{v}=\mathbf{w}$, and the model passes to classical linear elasticity. When $\xi \neq 0$ constitutive equations (3) describe the local bends of a particle with linear size $2 r$.

At the contact of particles, stresses should obey the continuity conditions

$$
\begin{array}{ll}
t_{11}\left(C_{i+1 j}\right)=t_{11}\left(A_{i j}\right), & t_{12}\left(C_{i+1 j}\right)=t_{12}\left(A_{i j}\right), \quad i=\overline{1, m-1}, \quad j=\overline{1, n} \\
t_{22}\left(D_{i j+1}\right)=t_{22}\left(B_{i j}\right), & t_{21}\left(D_{i j+1}\right)=t_{21}\left(B_{i j}\right), \quad i=\overline{1, m}, \quad j=\overline{1, n-1}
\end{array}
$$

When describing kinematic characteristics at contacts, it is possible to model normal and shear displacement discontinuities, which enables description of dilatancy, internal friction, cohesion and plastic shearing in a geomedium, including its strength loss [10]. This 
study is limited to the simplest variant of particle interaction and assumes continuous displacement at the grain boundaries, i.e.

$$
\begin{aligned}
& u_{1}\left(C_{i+1 j}\right)=u_{1}\left(A_{i j}\right), u_{2}\left(C_{i+1 j}\right)=u_{2}\left(A_{i j}\right), \quad i=\overline{1, m-1}, \quad j=\overline{1, n}, \\
& u_{1}\left(D_{i j+1}\right)=u_{1}\left(B_{i j}\right), u_{2}\left(D_{i j+1}\right)=u_{2}\left(B_{i j}\right), \quad i=\overline{1, m}, \quad j=\overline{1, n-1} .
\end{aligned}
$$

Now we are going to consider a boundary problem based on model (1)-(5).

\section{Numerical simulation}

We take the problem on deformation of rock mass in the vicinity of an extended horizontal opening in the conditions of generally tectonic natural stress field. One of the sources of higher rockburst hazard in mines in Western Siberia is the mixed gravity-and-tectonics type of the natural stresses in rock mass [15-17]. Some reference sources tell that the tectonic/gravity stress ratio ranges as $\lambda=0.5 \div 3$ and can sometimes be $\lambda=5$ and higher. In the test modeling of nonuniform compression of a rock sample with a circular hole [18], it is shown that tensile fractures appear at the hole boundary in this case. On the whole, the gravity-and-tectonic type of natural stress state means that zones affected by high stress gradients are generated in adjacent rock mass, which drastically impairs the mining safety. With all these taken into account, we solve the problem using the gradient model with structural parameter (1)-(5).

Let an extended horizontal opening have a radius $R$ and surrounding rock mass have linear sizes $L_{1}, L_{2}$ (plain strain deformation). The computational domain is a periodic lattice (Fig. 2). The natural gravity-and-tectonic stress field is modeled by the compressive forces $q, p$ set at the boundaries of the computational domain so that the lateral earth pressure coefficient is $\lambda=p / q$. The opening boundary is assumed to be stress-free. The boundary conditions are given by

$$
\begin{aligned}
& \left.t_{11}\left(C_{1 j}\right)\right|_{\Gamma_{1}}=\left.t_{11}\left(A_{m j}\right)\right|_{\Gamma_{1}}=-p,\left.\quad t_{12}\left(C_{1 j}\right)\right|_{\Gamma_{1}}=\left.t_{12}\left(A_{m j}\right)\right|_{\Gamma_{1}}=0, \quad j=\overline{1, n} \\
& \left.t_{22}\left(D_{i 1}\right)\right|_{\Gamma_{2}}=\left.t_{22}\left(B_{i n}\right)\right|_{\Gamma_{2}}=-q,\left.\quad t_{21}\left(D_{i 1}\right)\right|_{\Gamma_{2}}=\left.t_{21}\left(B_{i n}\right)\right|_{\Gamma_{2}}=0, \quad i=\overline{1, m} \text {. }
\end{aligned}
$$

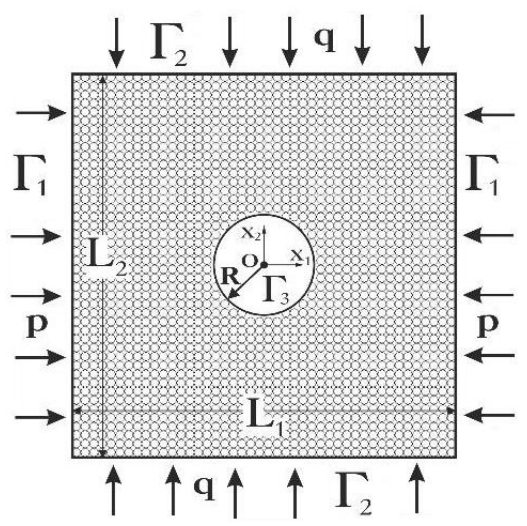

Fig. 2. Computational domain of rock mass in the vicinity of underground opening. 
The boundary $\Gamma_{3}$ is curved. It is approximated with a broken line containing only horizontal and vertical contacts. The sets of such contacts are denoted as $\Gamma_{3}^{\text {horiz }}$ and $\Gamma_{3}^{\text {vert }}$, i.e. $\Gamma_{3}=\Gamma_{3}^{\text {horiz }} \cup \Gamma_{3}^{\text {vert }}$ Correct conditions for equations (1)-(5) is setting either stresses $t_{21}, t_{22}$ or displacements $v_{1}, v_{2}$ at the contact set $\Gamma_{3}^{\text {horiz }}$, and either stresses $t_{11}, t_{12}$ or displacements $u_{1}, u_{2}$ at the contact set $\Gamma_{3}^{\text {vert }}$. Naturally, more complicated conditions are also possible. Thus, the condition of the stress-free boundary of the underground opening takes the form

$$
\begin{aligned}
& \left.t_{21}\right|_{\Gamma_{3}^{\text {horiz }}}=\left.t_{22}\right|_{\Gamma_{3}^{\text {horiz }}}=0, \\
& \left.t_{11}\right|_{\Gamma_{3}^{\text {vert }}}=\left.t_{12}\right|_{\Gamma_{3}^{\text {vert }}}=0 .
\end{aligned}
$$

Equations (1)-(7) constitute a closed system of equations for the unknown stresses and displacements determined at the points of the two above-mentioned difference meshes. This system is solved using the Gaussian method with selection of a principal element in a row of the matrix.

We select the values of the involved parameters as follows

$$
p=\lambda, q=1, E=2000, \mu=0.25, L_{1}=L_{2}=3.85, \quad R=1, r=0.04 .
$$

Here, all values of the length dimension are referred to $R$, and the values of the stress dimension are referred to $q$. We vary two parameters: the structural parameter $\xi$ (its dimension $\left[\mathrm{m}^{2} / \mathrm{Pa}\right]$ is referred to $R^{2} / q$ ) and the dimensionless lateral earth pressure coefficient $\lambda$.

The basic solution is selected as the classical elasticity solution. First, we perform calculation for $\xi=10^{-12}$, i.e., actually for $\xi \rightarrow 0$, when the solution tends to the linear elasticity solution. Figure 3 shows the contour lines of the maximum shear stress $T=0.5 \sqrt{\left(t_{11}-t_{22}\right)^{2}+4\left(\left(t_{12}+t_{21}\right) / 2\right)^{2}}$ at different $\lambda$. It is evident that the increase in the ratio of the tectonic stresses from $\lambda=1$ to $\lambda=3$ over the gravity stresses results in the concentration of the maximum stresses at the points nearby the roof and floor of the underground opening. In the theory of elasticity, this is a classical result.

a)

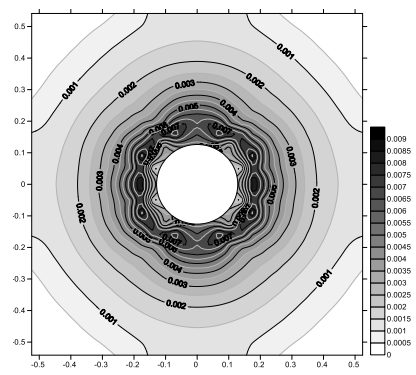

b)

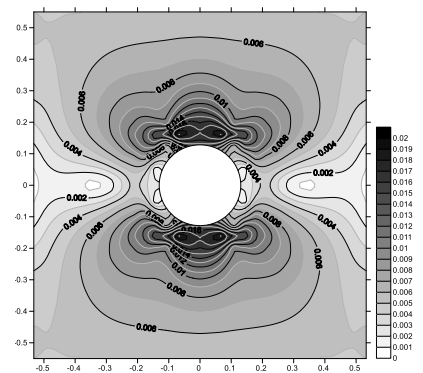

c)

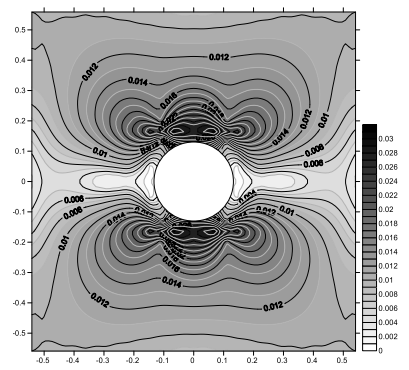

Fig. 3. Contour lines of $T=0.5 \sqrt{\left(\sigma_{x x}-\sigma_{y y}\right)^{2}+4 \sigma_{x y}^{2}}$ at different values of the structural parameter $\xi=10^{-12}$ : a) $\lambda=1$; b) $\lambda=2$; c) $\lambda=3$. 
With a gradual increase in the value of the structural parameter $\xi$, the higher stress concentration zones shape along the straight lines parallel to the coordinate axis and embrace an increasingly larger area of rock mass in the vicinity of the underground opening. Figure 4 depicts the calculations at $\xi=10^{-5}$. The contour lines become angular, and the maximum stress concentration domains propagates in-depth of rock mass and mostly in the horizontal direction, for a distance exceeding the opening size. Is is worthy of mentioning that the value of maximum stress concentration is somewhat lower in the latter case than in elastic rock mass. At $\lambda=3$ the reduction is $10 \%$.

a)

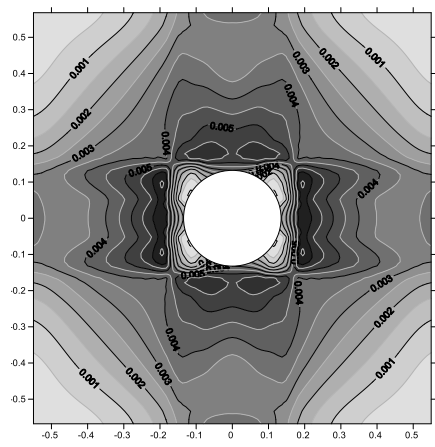

b)

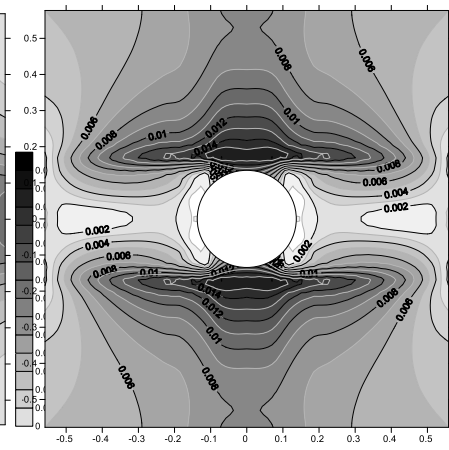

c)

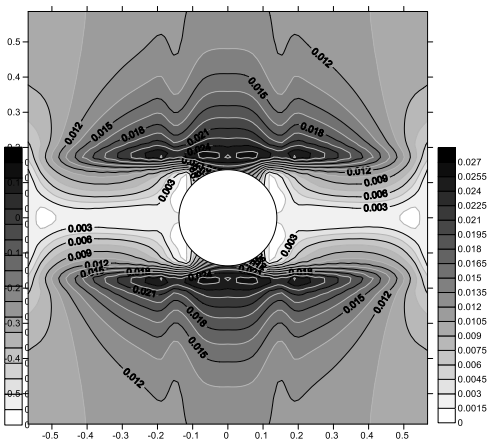

Fig. 4. Contour lines of $T=0.5 \sqrt{\left(\sigma_{x x}-\sigma_{y y}\right)^{2}+4 \sigma_{x y}^{2}}$ at different values of the structural parameter $\xi=10^{-5}$ : a) $\lambda=1$; b) $\lambda=2$; c) $\lambda=3$.

According to the numerical modeling in [19], in the conditions of increased rockburst hazard, in rock mass containing concentration zones of self-balancing stresses, displacement of loading from the boundary of a stope depthward adjacent rock mass leads to the release of energy of these stresses and can provoke jump-wise strength loss. Thus, it can be expected that enlargement of the influence zone of higher stress concentration (Fig. $4 \mathrm{c})$, even if its modulus is partly decreased, can provoke dynamic pressure events and initiate failure of mined-out areas.

\section{Conclusions}

1. The mathematical model with a structural parameter is adapted to the stress-strain analysis of rockburst-hazardous rock masses affected by high pressure gradients.

2. Essentially tectonic-type stress field contributes to the expansion of the higher stress concentration zones and provoke dynamic pressure events in rock mass.

The study was supported by the Russian Foundation for Basic Research (Project No. 20-0500184) and in the framework of the Basic Research project (Registration No. 121052500138-4).

\section{References}

1. V.V. Novozhilov. Two articles on mathematical models in continuum mechanics. (Inst. Probl. Mekh. AN SSSR Moscow, 1983)

2. I.I. Blekhman, A.D. Myshkis, Ya.G. Panovko. Mechanics and applied mathematics. 
Logic and applications in mathematics (Nauka - Fizmatgiz, Moscow, 1990)

3. V.A. Trofimov, Yu.A. Filippov. J. of Mining Science, 55 (5), $722-732$ (2019)

4. V.M. Seryakov, S.V. Rib, et al., J. of Mining Science, 54 (6), 899-906 (2018)

5. V.I. Klishin, G.Yu. Opruk, et al., J. of Mining Science, 56 (3), 395-403 (2020)

6. E. Cosserat, F. Cosserat. Theorie des corps deformables (Paris Hermann et fils, 1909)

7. W. Novatcki, Theory of micropolar elasticity (1970)

8. N.A. Fleck, J.W. Hutchinson, Adv. Appl. Mech, 33, 295-361 (1997)

9. I.Yu. Smolin, Modelir. Sistem Protsess, 14, 189-205 (2006)

10. A.F. Revuzhenko, O.A. Mikenina, J. Appl. Mech. and Techn. Phys., 59 (2), 332-340 (2018)

11. S.V. Lavrikov, A.F. Revuzhenko, AIP Conf. Proc., 1893 (2017)

12. V.I. Altukhov, S.V. Lavrikov, A.F. Revuzhenko, IOP Conf. Series: EES, 773, (2021)

13. P.V. Trusov, Mat. Modelir. Sistem Protsess, 17, 85-95 (2009)

14. A.F. Revuzhenko, S.V. Lavrikov, O.A. Mikenina, J. Fundament. Appl. Min. Sci, 7(1), 131-137 (2020)

15. A.V. Lovchikov, J. Fundament. Appl. Min. Sci., 4 (2), 105-111 (2017)

16. V.A. Eremenko, L.N. Gakhova, E.N. Semenyakin, J. of Mining Science, 48 (2), 269275 (2012)

17. D.V. Sidorov, M.I. Potapchuk, A.V. Sidlyar, Zap. Gorn. Inst., (Gorn. Delo), 234, 604611 (2018)

18. S.V. Suknev, J. of Mining Science, 56 (2), 174-183 (2020)

19. S.V. Lavrikov, A.F. Revuzhenko, J. of Mining Science, 56 (6), 887-902 (2020) 